\title{
Nasal Valve Lift in Nasal Valve Stenosis-A 2 Years Clinical Trial
}

\author{
Helen Heppt ${ }^{1}$ Julia Vent, MD² Mohammad Alali, MD ${ }^{3}$ Caroline Paal ${ }^{1}$ Werner Heppt, MD ${ }^{3}$ \\ 1 University of Ulm, Medical Faculty, Ulm, Germany \\ ${ }^{2}$ Department of Otorhinolaryngology, Ruprecht-Karls-University of \\ Heidelberg, Medical Faculty at Mannheim, Mannheim, Germany \\ ${ }^{3}$ Department of Otorhinolaryngology, Head and Neck Surgery, \\ Address for correspondence Werner Heppt, MD, Department of \\ Otorhinolaryngology, Head and Neck Surgery, Facial Plastic Surgery, \\ Klinikum Karlsruhe, Moltkestraße 90, 76133 Karlsruhe, Germany \\ (e-mail: wheppt@web.de).
}

Facial Plastic Surgery, Klinikum Karlsruhe, Karlsruhe, Germany

Facial Plast Surg 2019;35:14-22

\author{
Abstract \\ Keywords \\ - nasal valve stenosis \\ - nasal valve lift \\ - absorbable coiled PLA \\ threads \\ - nose surgery \\ - rhinoplasty
}

A clinical study was conducted to determine the effect of nasal valve lift treatment using absorbable, polylactic acid, self-retaining cone threads (Silhouette Soft) in patients with nasal valve stenosis. Sixty patients $(n=60)$ were included in this prospective study which was performed from January 2015 until December 2018, 19 patients dropped out due to noncompliance. Initially, participants were divided into two equal groups. One was treated by nasal valve lift only, the other additionally received turbinoplasty. A visual analog scale (VAS) was used to rate the sense of nasal ventilation. Patients' satisfaction was examined by a categorical scale, preoperatively, after 1 week, and after 1, 6, 12, 18, and 24 months. For statistical analysis, an univariant variance analysis was conducted to test the significance of differences in nasal breathing within one group at different points of postoperative controls, with the statistical significance set at $p<0.05$. Moreover, the two groups were compared with each other regarding results of the categorical scale. After surgical treatment, an improvement is reported by all participants. Average VAS scores were significantly lower between preoperative and every postoperative rating. Although a decline in the effect of breathing improvement is recognizable as threads are dissolved, patients' satisfaction in improvement of nasal breathing is persistent in most cases, even at long-term observation. Overall, these results demonstrate that valve lift is an effective method to treat patients with nasal valve stenosis.
The nasal valve area obviously is one of the most important anatomical areas of the upper respiratory tract, representing up to $80 \%$ of the nasal resistance. ${ }^{1}$ Located between the nostril and the nasal isthmus, it is the transition zone between the skin and the respiratory epithelium. The major part of the nasal valve area accounts for the alar region, mainly composed of the lower lateral cartilage and the septum. The isthmus-also known as the internal nasal valve-is the cranial border of the valve area to the nasal cavity, defined by the nasal septum and the caudal end of the upper lateral cartilage. With an average size of 50 to $70 \mathrm{~mm}^{2}$ and an angle of 10 to $15^{\circ}$ at its apex, it is the narrowest point of the upper respiratory tract acting as accelerator and diffusor of the inhaled air (Bernoulli's principle). ${ }^{2}$

Issue Theme New Tools and Proven Techniques in Rhinoplasty; Guest Editors: Enrico Robotti, MD, and Werner J. Heppt, MD
Concomitant to the airstream acceleration in the nasal isthmus with an air velocity of up to 12 to $18 \mathrm{~m} / \mathrm{s}$, there is a reduction in the static pressure resulting in a negative pressure and an inward traction of the nasal wings (Venturi effect). The valve area is very susceptible to changes. According to HagenPoiseuille law, even small constrictions lead to disproportionately high increases in the resistance. ${ }^{2,3}$ That is the case if any mucosal, skeletal, or cartilaginous deformities compromise the area. Caudal septal deflections, weakness, malformation, and malposition of the lower and upper lateral cartilages, ptotic tip deformities, and impaired nasal breathing by hypertrophic inferior turbinates belong to the most common causes of a valve dysfunction. ${ }^{3}$ According to estimates by clinicians, every second nasal obstruction is caused by nasal valve
Copyright (c) 2019 by Thieme Medical Publishers, Inc., 333 Seventh Avenue, New York, NY 10001, USA. Tel: +1(212) 584-4662
DOI https://doi.org/ 10.1055/s-0039-1677854. ISSN 0736-6825. 
stenoses, mostly by its internal component, leading to a disease-specific decrease in quality of life. ${ }^{4}$

As there exist various pathologies for nasal valve stenosis, the choice for a conservative or surgical treatment needs to be adjusted to the specific dysfunction or anatomical malformation. Nasal endoscopy, cone-beam computed tomography, photography, and airstream simulation ${ }^{5}$ are evaluated as useful diagnostic tools to detect abnormalities in the nasal anatomy, but not considered as reliable pre- and postoperative diagnostic tools. With the nasal valve stenosis mostly being described as a clinical diagnosis, assessment of nasal breathing using symptom grading and visual analog scales (VAS) has found to be the only appropriate parameters to impose the benefit of a valve treatment and patients' content. ${ }^{6}$ The aim of this study was to examine the outcome of nasal valve lift with absorbable, polylactic acid (PLA), selfretaining cone threads usually indicated for facial cosmetology. The technique first described by Saban et al in $2014^{7}$ presents a minimally invasive method to open the nasal valve, filling the gap between poorly tolerated external orthoses, internal stents and invasive surgical procedures requiring grafts or alloplastic materials. Nasal isthmus stenosis characterized by a reduced angle and weak alar or upper lateral cartilages with the tendency for an alar collapse are considered as pathologies well suited for a nasal valve lift treatment.

The present investigation was based on the pre- and posttherapeutic assessment of nasal breathing via symptom grading and VAS evaluating the effect on breathing improvements and patients' content. Information about the clinical outcome of patients treated with minimal invasive nasal valve lift may contribute to an optimized therapeutic standard in the treatment of nasal valve stenosis.

\section{Nasal Valve Lift Technique}

The nasal valve lift is based on the use of threads designed with cones in bidirectional orientation (-Fig. 1A). Pulling on both ends of the thread provides a suspension effect by hooking up the tissue on the cones in different directions (-Fig. 1B). The Silhouette Soft threads (Sinclair Pharma) utilized in the present study (-Fig. 1A) are absorbable, biocompatible, and biodegradable. The threads are made of PLA and the cones of polylactic-co-glycolic acid ( $82 \%$ of lactic acid, $18 \%$ of glycolic acid). The breakdown process of PLA by hydrolysing ester bonds and producing lactic acid, a naturally tissue substance, takes about 10 to 12 months. Silhouette Soft threads are not only used for repositioning of the skin and subcutaneous tissue but also lead to a fibroblast activation accompanied with an increased collagen production in the long run. ${ }^{8}$

In general, the nasal valve lift is performed under local anesthesia or under sedation, in a supine position, the upper body raised to $45^{\circ}$. After local disinfection of the skin and the nasal vestibulum with an Octenidindihydrochlorid-Phenoxyethanol solution (Octenisept; Schülke \& Mayr), local infiltration is applied on the nasion, the lateral nasal flank, and the isthmus area in the intercartilaginous zone with lidocaine $1 \%$ with adrenalin 1:100 000 . The skin in the nasion
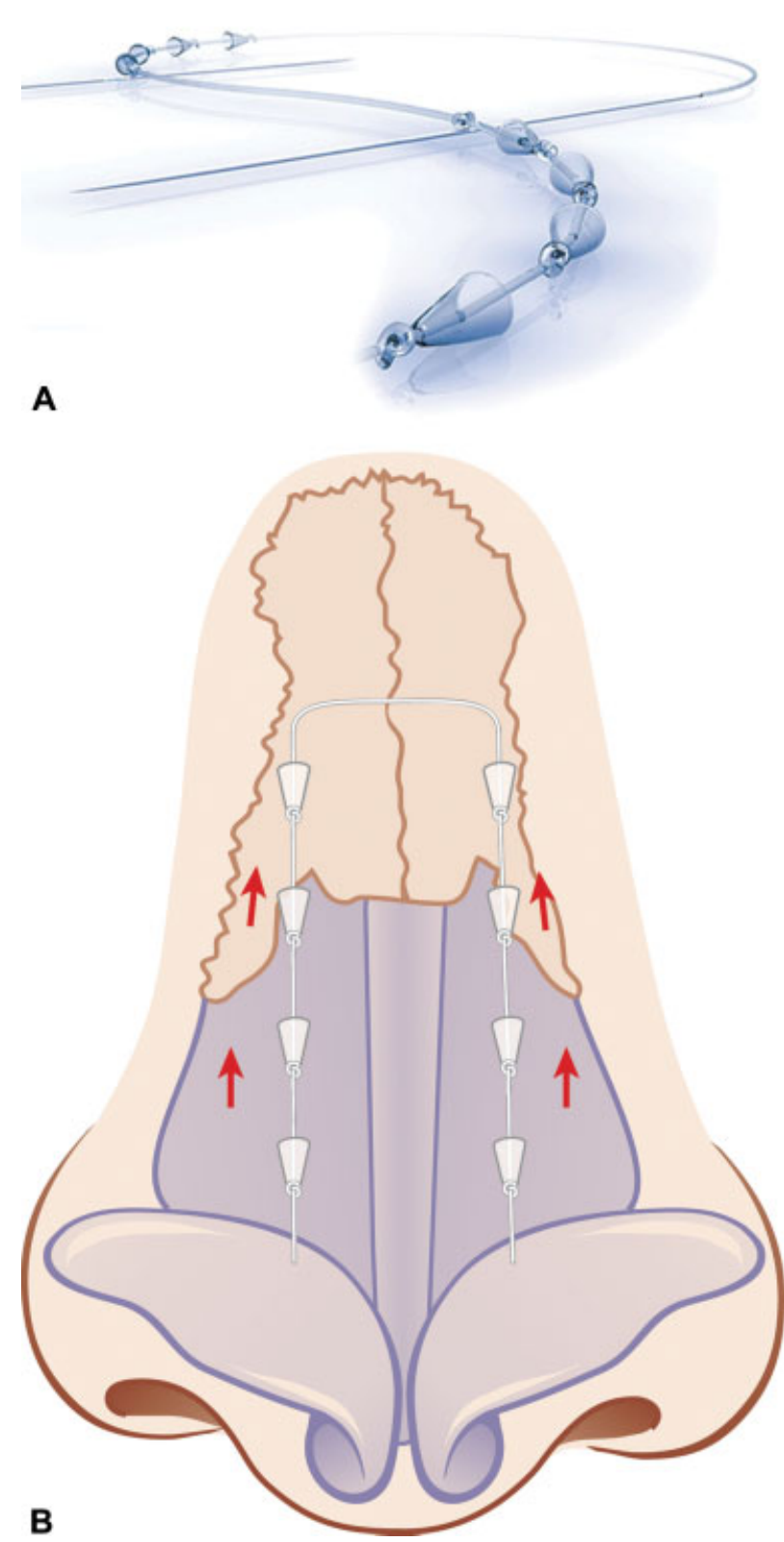

Fig. 1 Nasal valve lift technique. (A) Silhouette Soft thread with cones in bidirectional orientation (with permission of Sinclair Pharma). (B) Position of the thread in nasal valve lift procedure.

area is punctured with a 20-gauge yellow needle running just above the bone in the deep subcutaneous layer. Then the needle of the eight cone Silhouette Soft thread is inserted ( - Fig. 2A) and brought through the tissue under guidance of the canula (-Fig. 2B). After removal of the canula (-Fig. 2C), the first part of the thread containing four cones is brought out of the skin by pulling on it ( - Fig. 2D) so that the cone-free middle part of the thread is placed under the skin of the nasion. Next, the Silhouette Soft needle is re-entered at the thread's exit point (-Fig. 2E) and guided under digital control to the middle of the nasal isthmus passing in the subcutaneous plane right directly above the bony lateral nasal flank. Having passed the exit point in the valve area (-Fig. 2F), two cones are brought out by pulling on the thread 
A

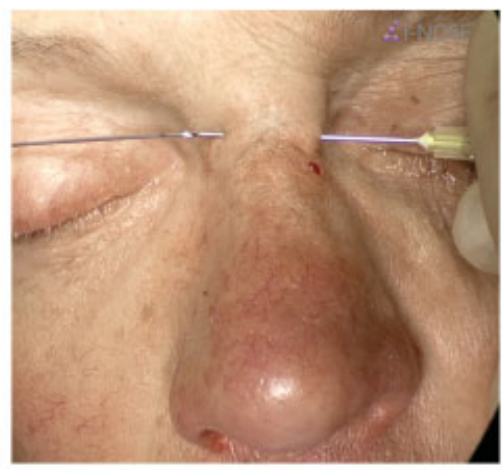

D

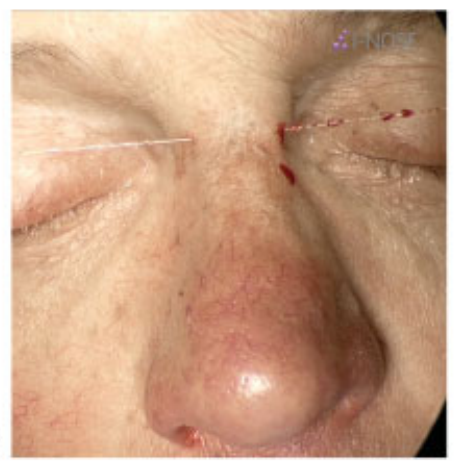

E

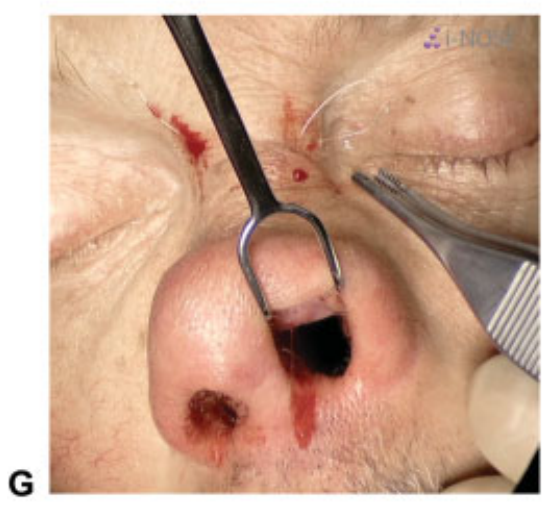

G

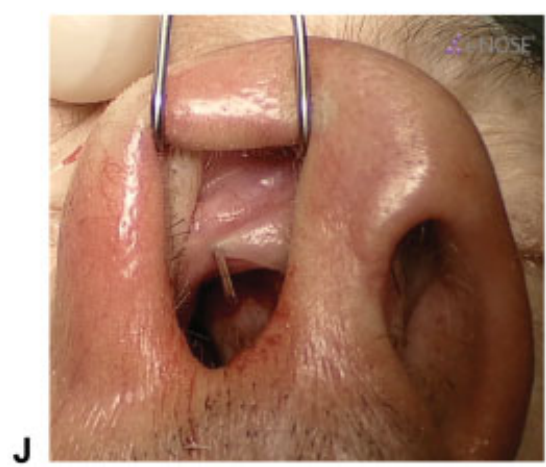

B
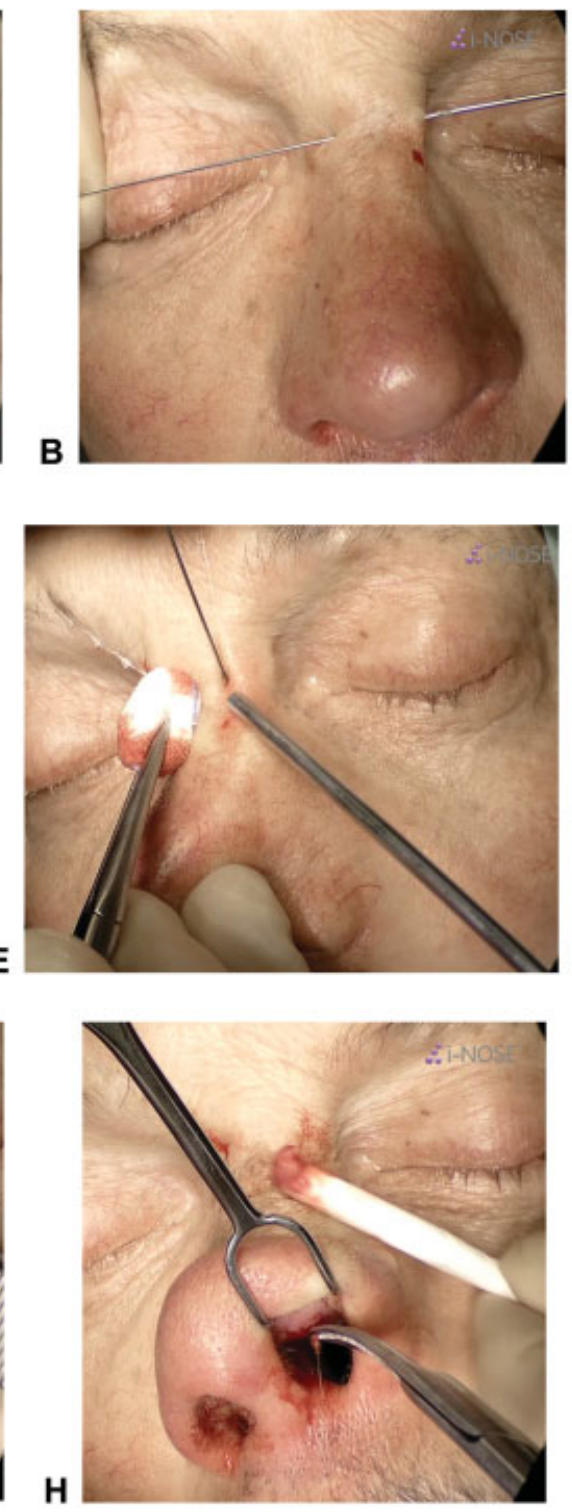

C

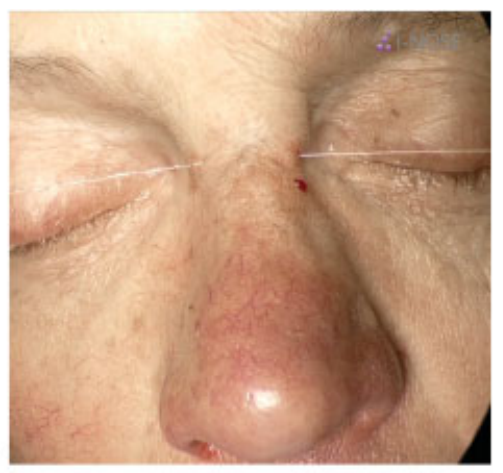

F
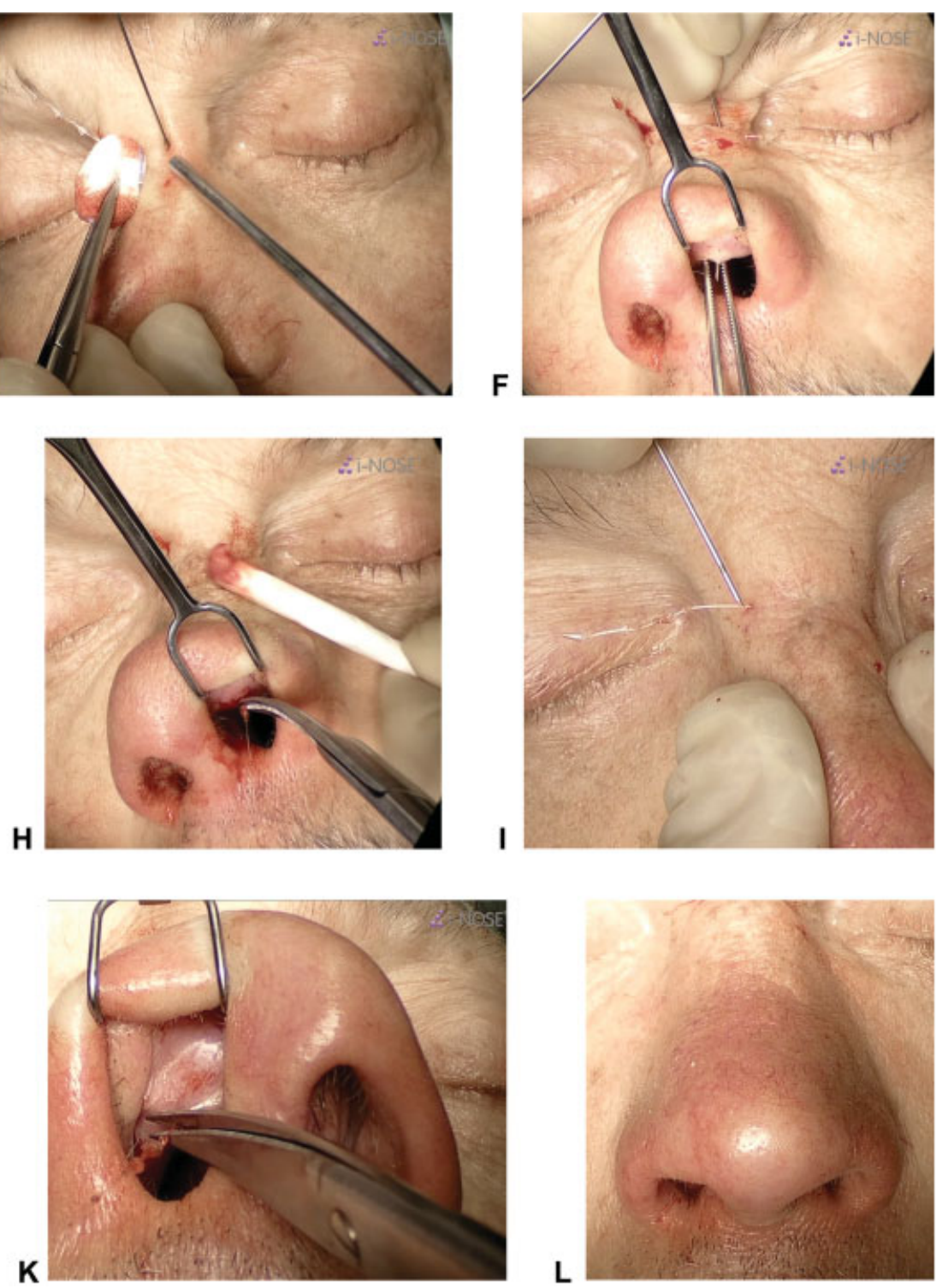

Fig. 2 Nasal valve lift technique using 8 Cone Silhouette Soft thread. (A) Skin puncture with a yellow needle and insertion of the suture needle. (B) The needle is brought through the tissue under guidance of the canula. (C) Removal of the canula. (D) Four cones of the thread are pulled through the skin. (E) Silhouette Soft needle reentered at the threads exit point. (F) Exit point of the needle in the middle of the nasal isthmus caudally to the upper lateral cartilage. (G) Cones are developed by redraping the vestibular skin. (H) Cut of the thread. (I) Reentry of the needle on the contralateral side. (J) The needle passes the exit point of the right isthmus. (K) Cut of the thread on the right. (L) Only slight swelling of the ala.

and redraping the vestibular skin (-Fig. 2G). Dragging the skin of the vestibule and the lateral nasal flank in an upward direction, the nasal isthmus and the lateral soft tissue will be hooked on the cones and pulled up in a superior-lateral vector. The thread is cut off on the first side (-Fig. $\mathbf{2 H}$ ) and the second needle finally brought in in the same way on the 
contralateral side with entry in the nasion ( - Fig. 2 I) and exit in the middle of the isthmus area at the caudal margin of the upper lateral cartilage (-Fig. 2J). The procedure is finished after the second suture has been developed and cut as described (-Fig. 2K). At the end of the procedure, which takes $\sim 20 \mathrm{~min}$, there is no disfiguration, except for a slight swelling of the ala (-Fig. 2L). Spontaneous closure of the vestibular skin obviates the need for suturing. The postoperative setting consists of cooling the nose for the first 2 hours and by application of an adhesive tape around the tip of the nose and on the nasion to sustain the upward pull effect and cover the entry points. To prevent local infections in the vestibulum, the patients are told to apply an antibiotic ointment (Infectogenta; Infectopharm) once daily for 1 week. To reduce dysesthesia at the lateral nasal wall, glasses should not be worn during that time.

\section{Patients and Methods}

A prospective study with 60 patients $(n=60)$ was performed from January 2015 until December 2018. Patients included were Caucasians older than 18 years. In the history, they suffered from nasal obstruction with an insufficient treatment by steroids, local decongestants, or previous surgical procedures. For patients to be included in the study, the physical examination had to show a bilateral isthmus stenosis (-Fig. 3), a positive Curette test, a positive Cottle maneuver, and alar collapse while forced inspiration. Additionally, hyperplasia of the turbinates was accepted as inclusion criterium. Previous surgery in the nasal valve area, known adverse reactions to threads or fillers, autoimmunologic diseases, acute or chronic sinonasal infections or other severe nose problems such as acute allergic symptoms in case history were defined as exclusion criteria. Anatomical abnormalities such as marked septal deviation, nasal tumors, cicatricial valve stenosis, or nasal polyposis examined in nasal endoscopy were also to be debarred. All patients were re-evaluated immediately before the treatment. Preoperatively, an informed written consent about the off-label use of Silhouette Soft threads for nasal valve lift, the technical procedure, complications, and postoperative care were gath- ered and in the group of concomitant turbinoplasty for the radio frequency treatment of intranasal turbinates.

The selected 60 participants were divided into two groups containing 30 patients each. One group of patients, whose nasal obstruction was mainly caused by bilateral isthmus stenosis, received a nasal valve lift with PLA threads (Silhouette Soft, 8 cones, Sinclair Pharma) alone. The other group of 30 patients-who were additionally showing hyperplasia of the inferior turbinates-was treated with nasal valve thread lift and reduction in the inferior turbinates bilaterally via radio frequency technique at the same session (CURIS $4 \mathrm{MHz}$ Technology, Sutter Medizintechnik GmbH).

A Nasal Questionnaire Score according to Haye et $\mathrm{al}^{9}$ was completed ( - Fig. 4). With the study focusing on the change of breathing in patients treated with nasal valve lift or nasal valve lift plus turbinoplasty, the sense of obstruction needed to be objectified. For rating, a VAS ranging from 1 to 10 was documented. Since the scale counted $10 \mathrm{~cm}$ in total, one step was defined as $1 \mathrm{~cm}$. One was defined as open nasal ventilation and 10 as highly impaired nasal breathing. Preoperatively, patients were instructed to mark the scale. When rating their sense of obstruction in nasal breathing at less than 4, they were excluded from the study. Twenty-five healthy volunteers served as control group of the VAS. To identify changes of nasal breathing due to surgery, sense of obstruction was rated by VAS the day before operation, 1 week and $1,6,12,18$, and 24 months postoperatively. Additionally, a categorical scale was used. Patients were asked whether nasal breathing had improved completely, substantially, or mildly, was unchanged or worse compared with the preoperative situation (-Fig. 4).

\section{Statistical Analysis}

For statistical analysis, ${ }^{10}$ an univariant variance analysis (ANOVA) was conducted. The aim of the test was to determine the effect of an independent on a dependent variable. An ANOVA allows to test the significance of differences between more than two groups, with the significance level set at $p<0.05$. Therefore, arithmetic means need to be compared. In this study, average VAS scores for the sense of nasal obstruction within one group were analyzed. Conditions for performing an ANOVA were given. Normally

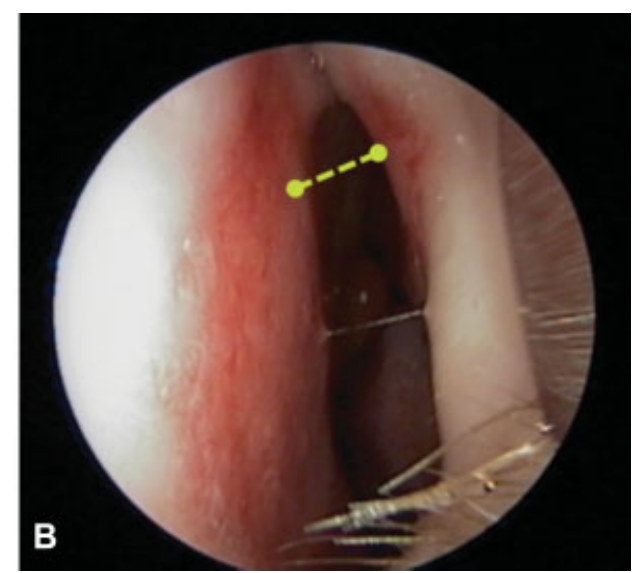

Fig. 3 Endoscopic view of the nasal isthmus. (A) Reduced angle in a patient with isthmus stenosis (array). (B) Isthmus angle of a healthy person (dotted line).

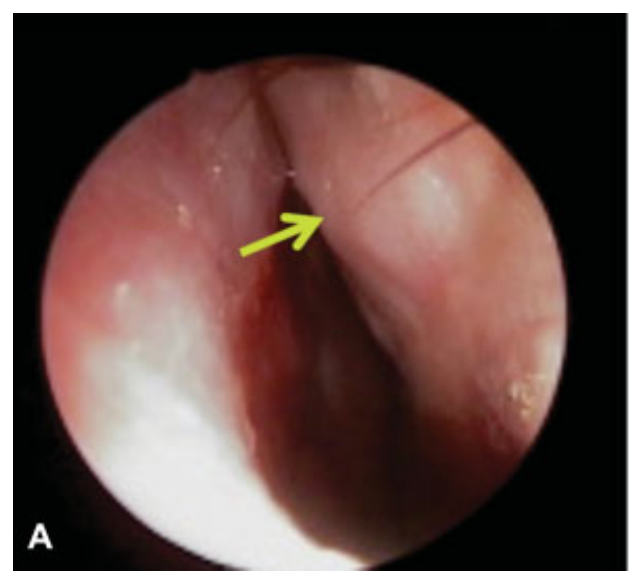




\title{
Nasal Valve Lift Questionnaire
}

\author{
Patient Initials: __ _ $\quad$ Date of birth: __ $\ldots+-{ }_{-}$Sex: _female _male \\ Rate your sense of obstruction (before, 1 week, 1, 6, 12, 18, 24 months after)
}

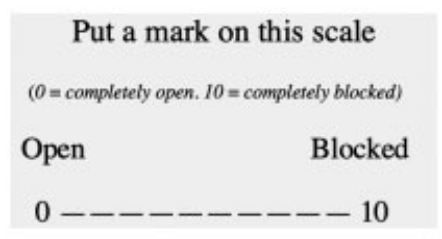

Specific postoperative questions (1 week, 1, 6, 12, 18, 24 months after)

Is Your Nasal Breathing

$\square$ Completely improved $\square$ Substantially improved $\square$ Mildly improved $\square$ Unchanged $\square$ Worse

Fig. 4 Nasal questionnaire according to Haye. ${ }^{9}$

distributed data was investigated by a Shapiro-Wilk test. The lack of outliers was checked by illustrations in box-plots and variance homogeneity by the Levene test. With the ANOVA showing a significant result $(p<0.05)$, the groups are considered as significantly different. To differentiate between which groups the significant difference exists, a Tukey posthoc was conducted.

\section{Results}

During the study period of 24 months, the dropout rate because of noncompliance was 19 patients, with a total of 41 cases completing the study. After 2 years, the group treated only by nasal valve lift (V-group) contained 19 participants ( $n=19$, age $23-76$ years, mean 16.2 , standard deviation 42.6 , 9 females, 10 males), while 22 patients $(n=22$, age 19-68 years, mean 39.9, standard deviation 13.9, 10 females, 12 males) with hyperplasia of the turbinates who additionally underwent radio frequency turbinoplasty (valve lift plus turbinoplasty group [VT-group]) remained. The majority of patients included in the present study were treated under local anesthesia with $5 \mathrm{~mL}$ Xylocaine 1\%, only nine under additional intravenous sedation with propofol (Disoprivan; Aspen) and remifentanil (Ultiva; Aspen).

For indicating the average sense of obstruction of the normal population and definition of a solid and reliable inclusion criterion, the VAS was performed by 25 participants $(n=25$, age 22-59 years, mean 39.3, standard deviation 10.3, 12 females, 13 males) without any breathing problems. Thereby the arithmetic mean of nasal breathing on VAS was 0.84 .

\section{Results of V-Group: Patients Only Treated by Nasal Valve Lift $(n=19)$}

Preoperatively, no sense of obstruction was rated less than 5 on VAS. The maximum was selected twice $(n=2)$. The average rating of the preoperative sense of obstruction was 7.2. One week after surgery, the average nasal ventilation on VAS selfassessment was 2.3. During the study period, the arithmetic mean increased continuously up to 4.0 after 24 months.

To test significance, an ANOVA was conducted between the arithmetic means within the V-group. Significant differences in medical studies are indicated with a $p$-value lower than 0.05 (10).

The average nasal obstruction rated by VAS was significantly less at every time point of postoperative control compared with preoperative findings. The greatest difference of VAS mean values found was 4.8 between preoperative stenosis and week 1, and the smallest of 3.2 at the end of the study.

Additionally, sense of obstruction in week 1 differed significantly in 1.6 from week 24 (-Fig. 5).

Consecutively, results of the categorical scale in V-group were analyzed (-Fig. 6). An improvement in nasal breathing was noted 1 week after the valve lift in every patient. In the long term, there was a decrease in the positive effect, but even after 2 years only a few rated the effect as unchanged compared with the preoperative status none as worsened.

This means in proportional terms that at the first postoperative control after 1 week, "completely improved" was selected by $26.3 \%$, "substantially improved" by $52.6 \%$, "mildly improved" by $21.1 \%$. No patient reported an unchanged sense of obstruction or a worsening in nasal breathing postoperatively. During the course of the study, the percentage of patients feeling "completely improved" consistently dropped down to $5.3 \%$ after 2 years. After a peak to $63.2 \%$ after 1 week, the selection of "substantially improved" also decreased to $31.6 \%$ after 24 months.

In contrast, the categories "mildly improved" and "unchanged" increased toward the end of the study. Two years after the therapeutic treatment, $47.7 \%$ of the participants sensed nasal obstruction as "mildly improved" and $15.8 \%$ as 


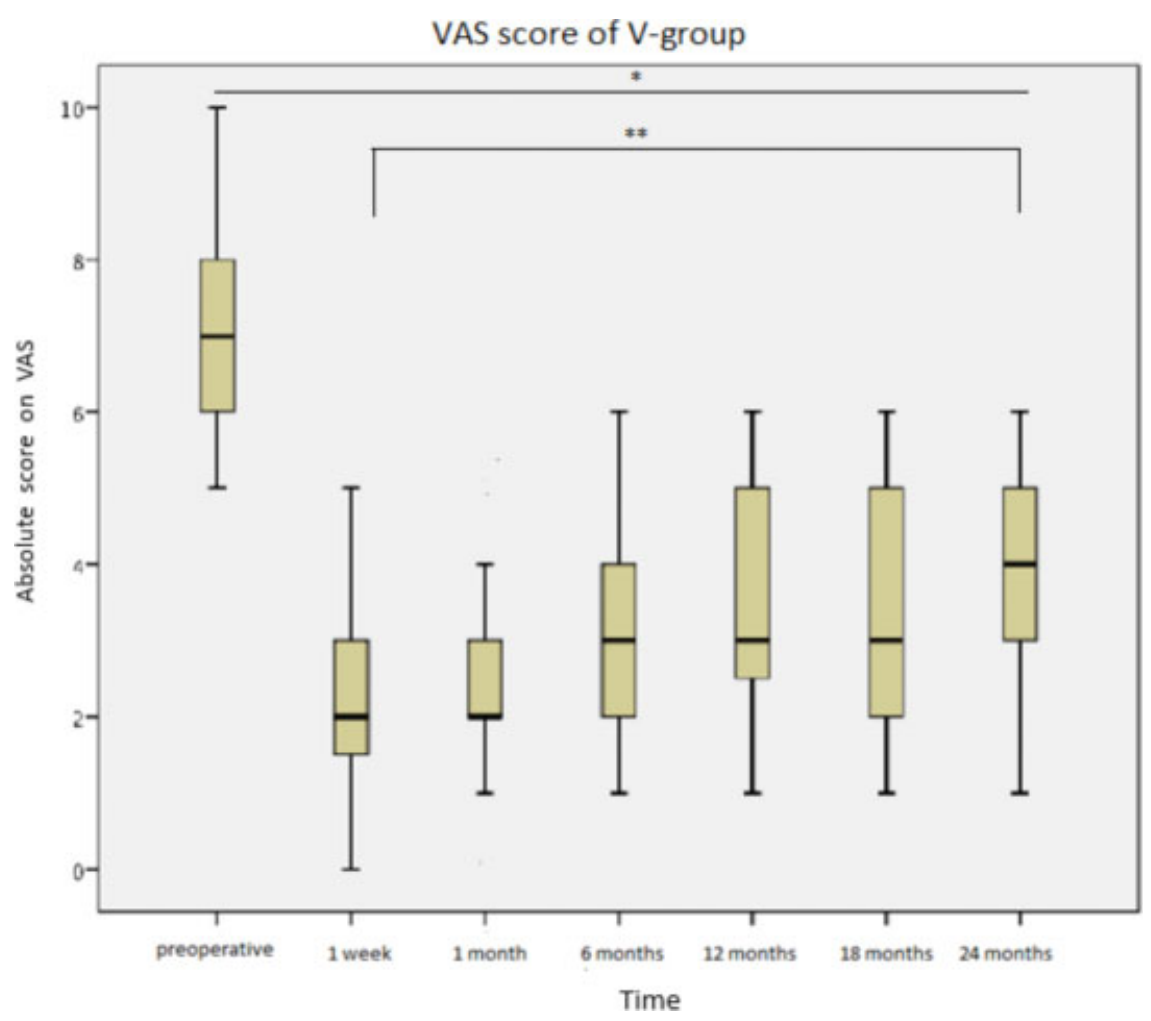

Fig. 5 Statistical analysis (univariant variance analysis) of absolute visual analog scale (VAS) values of patients treated only by nasal valve lift (V-group, $n=19$ ) preoperative, after 1 week, 1, 6, 12, 18, and 24 months. The boxplot represents quartile one, median, and quartile three. Whiskers are indicated for 1.5 interquartile range. Significant differences are indicated with $p<0.05$. $\left({ }^{*}\right)$ declares significance of groups that are reached by the horizontal line. The end of the horizontal line of $\left(^{* *}\right)$ marks significance between these two points of time.

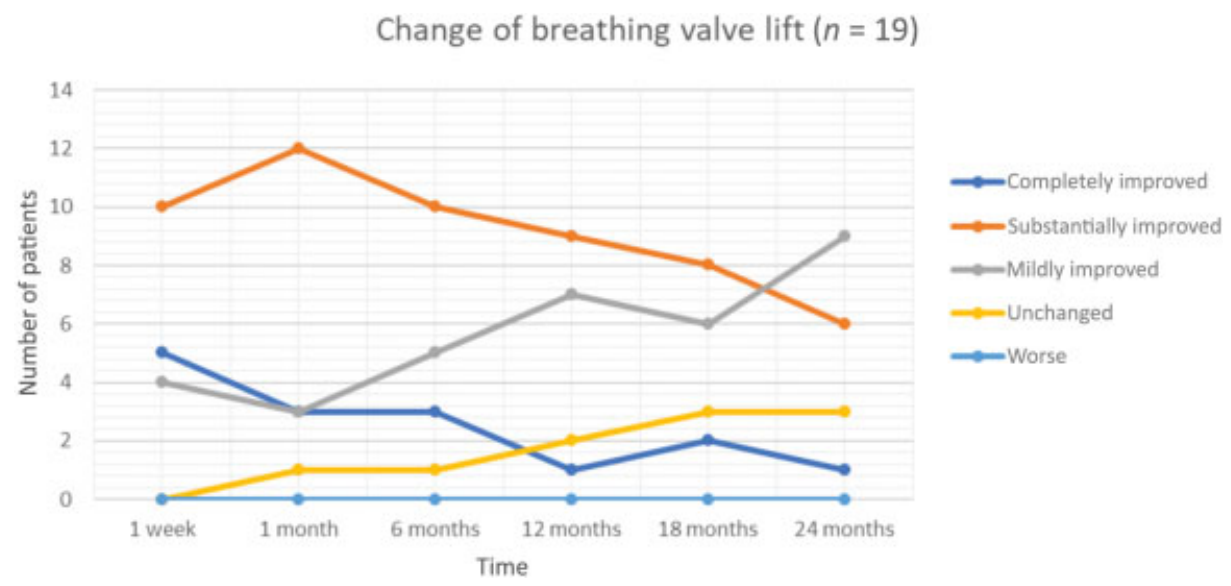

Fig. 6 Change of breathing after valve lift $(n=19) ; 1$ week, 1, 6, 12, 18, and 24 months postoperatively.

unchanged. None of the V-group patients reported a worsening at any time of postoperative controls.

\section{Results of VT-Group: Patients Treated by Nasal Valve Lift Plus Radio Frequency Turbinoplasty $(n=22)$}

Before the operation and at the first follow-up control after 1 week, ratings on the VAS were similar to those of the Vgroup. Nasal obstruction was never scaled less than 5 on VAS. The preoperative average rating of the sense of obstruction was 7.2. One week postoperatively, the arithmetic mean was 2.4 , whereas after 4 weeks it decreased to 1.5 . Two years after the operation, the average sense of obstruction was three.
To test significance, an ANOVA is conducted between the arithmetic means within VT-group with a $p$ value lower than 0.05 for significance. As in the V-group, the average sense of obstruction of all postoperative controls was significantly lower compared with preoperative findings ( - Fig. 7 ). The greatest difference of 5.8 was seen between average preoperative obstruction and 4 weeks postoperatively, the smallest after 18 months. Moreover, results 1 month after the intervention were significantly lower compared with the ones in month 18 and month 24 .

Results of the categorical scale in VT-group ( - Fig. 8) essentially followed a similar trend as compared with the V-group. 


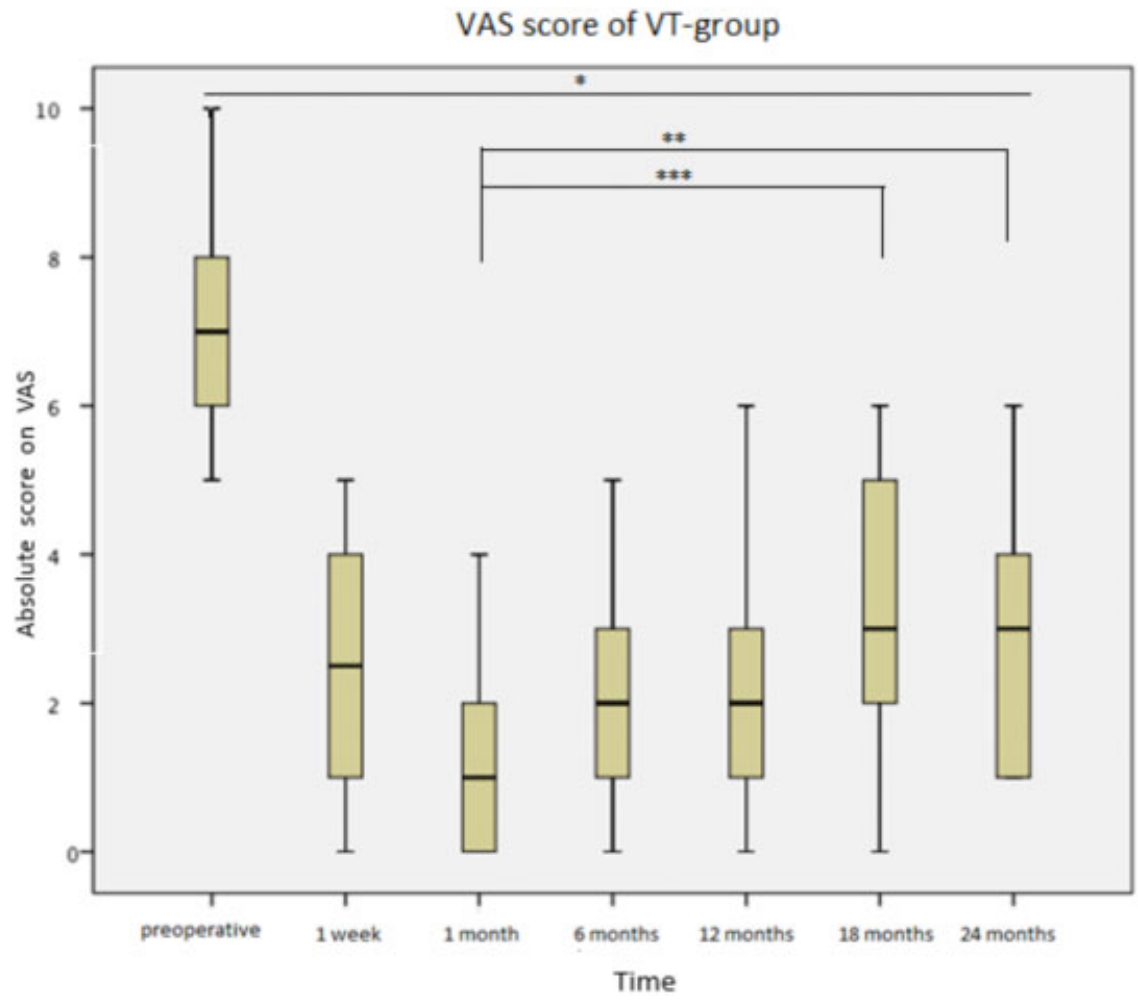

Fig. 7 Statistical analysis (univariant variance analysis) of absolute visual analog scale (VAS) values of patients treated by nasal valve lift and turbinoplasty (VT-group) preoperative, after 1 week, 1, 6, 12, 18, and 24 months. The boxplot represents quartile one, median, and quartile three. Whiskers are indicated for 1.5 interquartile range. Significant differences are indicated with $p<0.05$. $\left({ }^{*}\right)$ declares significance of groups that are reached by the horizontal line. The end of the horizontal line of $\left({ }^{* *}\right)$ and $\left({ }^{* * *}\right)$ marks significance between these two points of time.

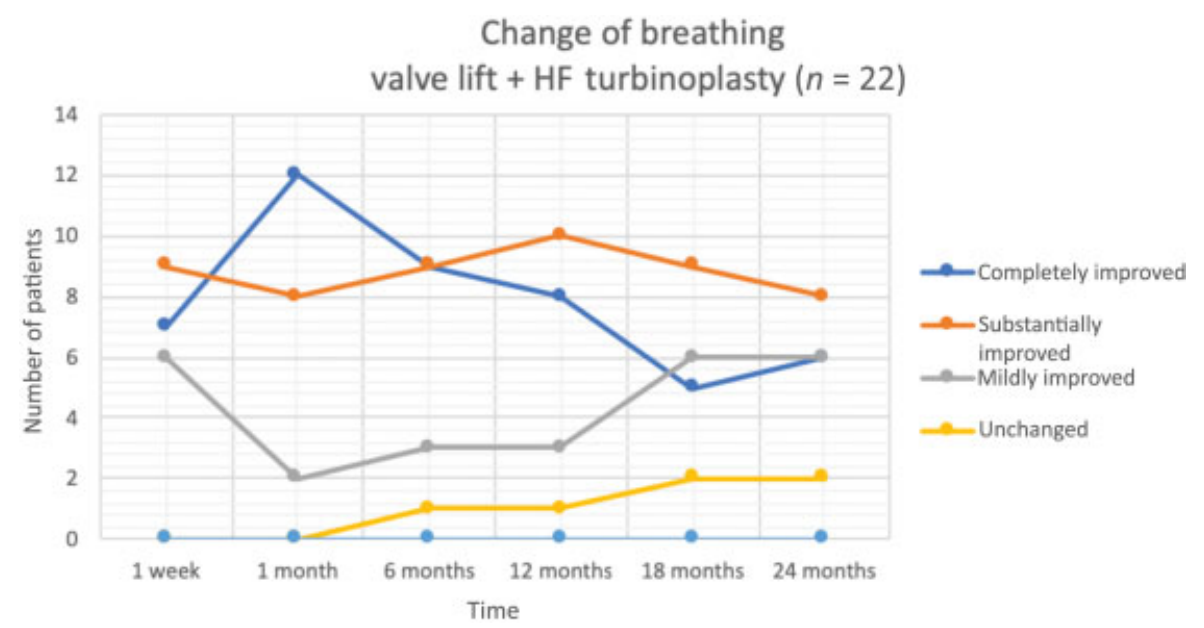

Fig. 8 Change of breathing after valve lift and turbinoplasty $(n=22) ; 1$ week, 1, 6, 12, 18, and 24 months postoperatively.

A more detailed analysis revealed a delayed effect of improvement in nasal breathing shifted from 1 week after the treatment(V-group) to 4 weeks and a generally higher improvement in the long run. Expressed in figures of percentages at the first postoperative control after 1 week, the sense of obstruction is in $31.8 \%$ of cases "completely improved," in $40.9 \%$ "substantially improved," and in 27.3\% "mildly improved." As in V-group, at this point of time no obstruction is estimated as "unchanged" or "worse." During the course of this study, a peak up to $54.4 \%$ of the category "completely improved" was evident after 1 month.
Thereupon, it dropped consistently down to $27.4 \%$ after 24 months. Throughout the study, the percentage "substantially improved" nasal breathing was approximately equal. As an increase in complete improvements was seen 4 weeks postoperatively, the selection of "mildly improved" inversely decreased to $9.1 \%$ at this point of time and arose to $27.3 \%$ after 2 years. An increase toward the end of the study was also seen in "unchanged" nasal breathing, counting 9.1\% after 24 months. In the V-group, none of the VT patients reported a worsening at any time of postoperative controls. 


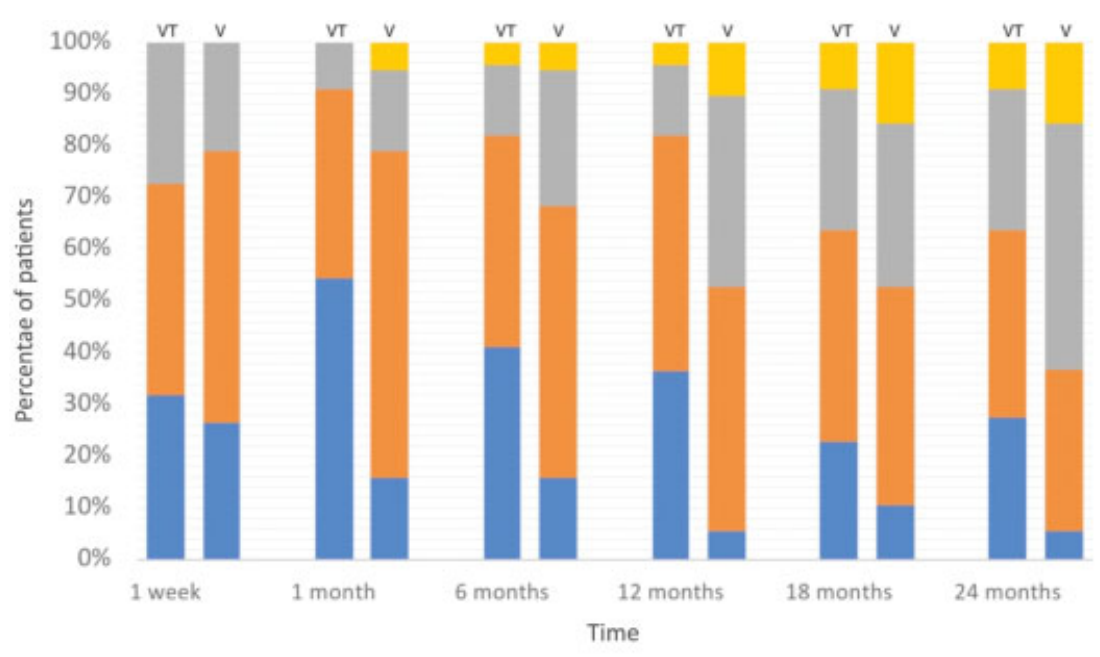

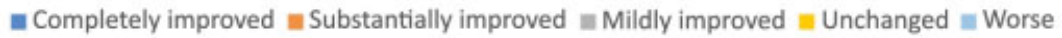

Fig. 9 Comparison of the categorical scale of the degree of change of nasal breathing in patients after treatment with nasal valve lift and turbinoplasty (VT-group, $n=22$ ) and only with nasal valve lift (V-group, $n=19$ ).

\section{Differences between V-Group and VT-Group}

-Fig. 9 shows the progression of the VAS values of both investigated patient groups. As previously mentioned, there is a parallel between both courses, with a delayed, but somewhat stronger therapeutic effect of the VT-group along the whole study period.

\section{Discussion}

Nasal valve stenosis is mostly described as a clinical entity with a lack of diagnostic and therapeutic standards. ${ }^{11}$ In January 2012, a new surgical treatment was invented. ${ }^{7}$ By using absorbable threads normally indicated for facial rejuvenation, an alternative to conservative valve treatment with tapes, stents or surgical grafts procedures, was created. ${ }^{12-14}$ The so-called nasal valve lift ranks as a minimal invasive procedure of short duration, 20 minutes on average. According to the literature, ${ }^{7}$ the applied Silhouette Soft threads are well tolerated with a low downtime postoperatively and low risks of side effects. Nasal ecchymosis, redness, discomfort wearing glasses, unpleasant sensation, swelling of the nasal flank, pain, dimpling, and entry point infection are reported, but only to a lesser extent and as a transient event of short duration. ${ }^{7}$ This present clinical trial was realized over an observational period of 2 years to evaluate the long-term effect of nasal valve lift on nasal obstruction caused by bilateral isthmus stenosis. Moreover, it was conducted to elaborate the outcome of a combination of nasal valve lift and turbinoplasty.

The assessment of changes of nasal breathing was based on subjective sensations of the participants. As diagnostic tools for objective measurements of nasal valve stenosis are still not standardized, ${ }^{11}$ patients' satisfaction was used to scale the clinical benefit of nasal valve lift. Advantages of using VAS as a measurement tool are an easy-handling, low susceptibility to errors and high resolution to differentiate symptoms that appear in a continuum. ${ }^{15}$ Though the VAS compared with other scales types such as the Likert scale is less vulnerable to bias referring to patients' satisfaction surveys, ${ }^{16}$ some systematic tendency has to be considered which is called the halo effect. Patients tend to select scores toward the middle of the scale and avoid extremes. Values of the VAS might be smaller than in categorical scales. ${ }^{16}$ Thus, a combination of VAS with a categorical scale is recommended and was selected for this study.

A look at overall results indicated valve lift as an effective method to treat patients with nasal valve stenosis. Preoperatively, the average sense of obstruction in all patients analyzed by VAS was ranked as $~ 70 \%$ blocked. After surgical treatment, an improvement was reported by all subjects. Slightly more patients treated by nasal valve lift alone felt "completely improved" after 1 week. Three weeks later, additional turbinoplasty influenced participants' content significantly. More than $50 \%$ sensed that their nasal breathing had completely improved. This result correlates to the average feeling of nasal obstruction selected on the VAS being the lowest throughout the whole study period. Obviously due to the mucosal swelling in the first days after radio frequency turbinoplasty, the highest levels of optimizing nasal breathing were reached only after 4 weeks compared with 1 week after exclusive valve lift. The long-term effect of submucosal turbinoplasty in addition to valve lift explains why the sense of obstruction is rated less by the group of patients who received both treatments, throughout the whole study period.

Obviously, the success of treatment dropped in both groups toward the end of the 2 years clinical trial. This occasion might be due to the resorption of the PLA threads after 9 to 12 months ${ }^{8}$ presuming a weak point of Silhouette Soft threads. Consequently, a revision of minimal-invasive nasal valve lift might be indicated. Nevertheless, even at the end of the clinical trial there was no complete decline of the treatment success which might be due to an induction of a collagen production while the thread resorption process. Throughout the 2 years, nasal breathing was never reported to be worse compared with preoperative findings at any point of postoperative control. 


\section{Conclusion}

Nasal valve stenosis is considered a pathology well suited for a nasal valve lift treatment based on the use of absorbable, PLA, self-retaining cone threads. Throughout the observation period of 24 months, an improvement in nasal breathing was seen in the total amount of 41 participants treated by nasal valve lift. Although a decline in the effect of breathing improvement is recognizable as threads resorbed, patients' satisfaction in changes of nasal breathing was persistent even until the end of the study. This minimally invasive method was herewith shown to be a valuable option in the treatment of nasal valve stenosis.

\section{Conflict of Interest}

None.

\section{References}

1 Wexler DB, Davidson TM. The nasal valve: a review of the anatomy, imaging, and physiology. Am J Rhinol 2004;18(03):143-150

2 Huizing $\mathrm{BH}$, de Groot JAM. Functional Reconstructive Nasal Surgery. 1 ed. New York, Stuttgart: Thieme; 2002

3 Heppt W, Vent J, Hildebrandt T. Nasal valve collapse. In: Ahmad Z, Morton R, Giles M, eds. Symptom oriented Otolarnygology Head and Neck Surgery. Rhinology and Facial Plastic Surgery. New Dehli: Jaypee; 2017, Vol 2:163-182

4 Rhee JS, Poetker DM, Smith TL, Bustillo A, Burzynski M, Davis RE. Nasal valve surgery improves disease-specific quality of life. Laryngoscope 2005;115(03):437-440

5 Hildebrandt T, Goubergrits L, Heppt WJ, Bessler S, Zachow S. Evaluation of the intranasal flow field through computational fluid dynamics. Facial Plast Surg 2013;29(02):93-98

6 Augé J, Vent J, Agache I, et al. EAACI Position paper on the standardization of nasal allergen challenges. Allergy 2018;73 (08):1597-1608
7 Saban Y, Javier B, Massa M. Nasal lift-nasal valve lift and nasal tip lift-preliminary results of a new technique using noninvasive selfretaining unidirectional nasal suspension with threads. Facial Plast Surg 2014;30(06):661-669

8 Athanasiou KA, Niederauer GG, Agrawal CM. Sterilization, toxicity, biocompatibility and clinical applications of polylactic acid/polyglycolic acid copolymers. Biomaterials 1996;17(02): 93-102

9 Haye R, Tarangen M, Shiryaeva O, Døsen LK. Evaluation of the nasal surgical questionnaire for monitoring results of septoplasty. Int J Otolaryngol 2015;2015:563639-563646

10 Fay DS, Gerow K. A biologist's guide to statistical thinking and analysis. WormBook 2013:1-54

11 Rhee JS, Weaver EM, Park SS, et al. Clinical consensus statement: diagnosis and management of nasal valve compromise. Otolaryngol Head Neck Surg 2010;143(01):48-59

12 Shemirani NL, Rhee JS, Chiu AM. Nasal airway obstruction: allergy and otolaryngology perspectives. Ann Allergy Asthma Immunol 2008;101(06):593-598

13 Khosh MM, Jen A, Honrado C, Pearlman SJ. Nasal valve reconstruction: experience in 53 consecutive patients. Arch Facial Plast Surg 2004;6(03):167-171

14 Spielmann PM, White PS, Hussain SS. Surgical techniques for the treatment of nasal valve collapse: a systematic review. Laryngoscope 2009;119(07):1281-1290

15 Klimek L, Bergmann KC, Biedermann T, et al. Visual analogue scales (VAS): measuring instruments for the documentation of symptoms and therapy monitoring in cases of allergic rhinitis in everyday health care: Position Paper of the German Society of Allergology (AeDA) and the German Society of Allergy and Clinical Immunology (DGAKI), ENT Section, in collaboration with the working group on Clinical Immunology, Allergology and Environmental Medicine of the German Society of Otorhinolaryngology, Head and Neck Surgery (DGHNOKHC). Allergo J Int 2017;26 (01):16-24

16 Voutilainen A, PitkäahoT, Kvist T, Vehviläinen-Julkunen K. How to ask about patient satisfaction? The visual analogue scale is less vulnerable to confounding factors and ceiling effect than a symmetric Likert scale. J Adv Nurs 2016;72(04):946-957 